\title{
Improving the Performance of Process Controllers Using a New Clustered Neural Network
}

\author{
M. Reza. Soleymani Yazdi ${ }^{1, a}$, Michel Guillot ${ }^{2, b}$ \\ ${ }^{1}$ Dept. of Mech. Eng., Imam Hussein University, Tehran - Iran \\ 2 Dept. of Mech. Eng., Laval University, Quebec - Canada \\ a mrsoley2002@gmail.com (Corresponding author) \\ b.michel.guillot@gmc.ulaval.ca
}

Keywords: Machining simulation; Process controller; clustered neural network

\begin{abstract}
This paper presents first a newly developed clustered neural network, which incorporates self-organization capacity into the well-known common multilayer perceptron (MLP) architecture. With this addition, it is possible to reduce significantly overall memory degradation of the neurocontroller during on-line training. In the second part of the paper, this clustered multilayer perceptron (CMLP) network is applied and compared to the MLP through modeling and simulations of machining processes. Simulation results presented using machining data demonstrate that the CMLP possesses more powerful modeling capacity than the standard MLP, offers better adaptability to new operating conditions, and finally performs more reliably. During on-line training with machining data about $65 \%$ degradation of previously learned information can be observed in the MLP as opposed to only $11 \%$ for the CMLP. Finally, an adaptive control scheme intended for on-line optimization of the machining processes is presented. This scheme uses a feed forward CMLP inverse neuro-controller which learns off-line and on-line the relationships between process inputs and output under simulated perturbations (i.e., tool wear and non-homogeneous workpiece material properties). The first results using the CMLP inverse neuro-controller are promising
\end{abstract}

\section{Introduction}

The need for intelligent and performing AC systems has led many investigators toward the development of more advanced schemes aiming at objectives such as:

- optimization of machining cost or

- improvement of part quality and more specifically surface finish and part dimensions.

Although considerable research effort and several schemes have been realized over the last 30 years $[1,2]$, their applicability and robustness for industrial machining control is still extremely limited for several reasons. First, most of these schemes are more complicated to set as they require from the technician of process planner time consuming calculations and inputs of lots of data in the controller. Second, for industrial use, a controller must adapt easily to various operations, cutting tools, part materials and geometry. Finally, with the complexity of most proposed AC schemes, it is almost impossible to ensure reliable performance in all conditions.

Considerable work has been realized on the development of AC systems for optimization using neural networks to achieve better performance and more robust controllers [3, 4 and 5]. Neural network models have been shown [2] to be superior to conventional polynomial and logarithmic modeling techniques. Over the last few years, multilayer perceptron (MLP) neural nets using online adaptation have been successfully applied in prototype AC systems [6] and have also been implemented and sold as an option in a commercial controller. An inverse process neuro-controller for optimizing productivity and part quality in turning has been presented [3]. An inverse process neuro-controller and a MLP neural network were applied to predict the cutting parameters for optimal process productivity and part quality. Hybrid neural networks have been recently applied to solve various types of machining problems [7, 8, 9 and 10]. Despite promising results, the degradation of previously learned knowledge is observed in neural network controllers when using 
on-line adaptation. A remedy is to provide a clustered structure to the network. In this way, only one cluster would be affected during on-line adaptation, thus reducing the risk of modifying unnecessarily previously memorized data.

\section{Clustered neural network}

The problem of degradation of stored information is strongly related to the nature of data processing in the artificial neural networks. Clustered networks appear as an interesting solution since only the cluster related to the exemplar is trained, leaving intact the rest of the network.

In the novel architecture of the CMLP, the neurons are no longer aligned, but are randomly placed in planes representing layers.

\section{Modeling performance of the clustered neural network Non-Linear Modeling and Simulation}

At this stage, the capacity for non-linear modeling and adaptability of the CMLP network is tested and compared to that of MLP network through off-line and on-line training respectively.

The precision and training speed of the CMLP network of dimensions $3 \times 15 \times 1$ are compared with those of the MLP network of same dimensions. The CMLP network is expected to show a greater resistance with respect to the memory degradation problem of the stored information. Consequently, this resistance will be qualified during on-line training.

From Fig. 1, it is clear that the best results are obtained with the CMLP networks. These latter gradually improve their performances with the sequential training of the new data. These performances are slightly degraded for the data learned a priori. About $11 \%$ and $65 \%$ memory degradation can be observed in CMLP and MLP respectively.

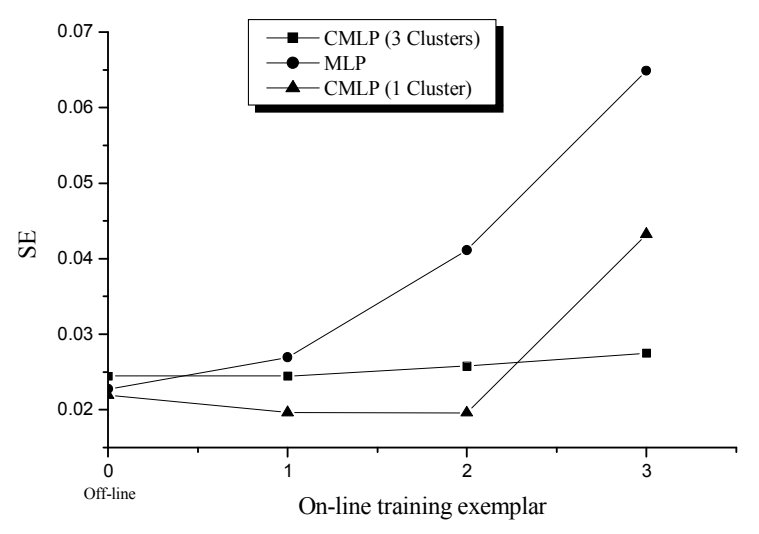

Figure 1 Effect of on-line training on overall modeling error

\section{Flexibility to Different Process Operations}

Two MLP and CMLP networks with different parameter settings are used to learn simultaneously the behavior of two milling operations i.e. peripheral cutting and slotting.

Theoretical cutting models of surface finish $\left(R_{a}\right)$ and cutting force components $\left(F_{x}, F_{y}\right)$ can be estimated by use of [5].

The cutting force components

$$
\begin{aligned}
F x= & K_{t} \cdot N_{f} \cdot f_{t} \cdot A_{d}\left\{K_{r}\left[\sin \left(2 \beta_{e n}\right)-2 \beta_{e n}\right]\right. \\
& \left.+\left[1-\cos \left(2 \beta_{e n}\right)\right]\right\} /(8 \pi) \\
F y= & K_{t} \cdot N_{f} \cdot f_{t} \cdot A_{d}\left\{K_{r}\left[1-\cos \left(2 \beta_{e n}\right)\right]\right. \\
& \left.+\left[2 \beta_{e n}-\sin \left(2 \beta_{e n}\right)\right]\right\} /(8 \pi)
\end{aligned}
$$

Surface finish for slot cutting

$R_{a}=\left(3.18 \times 10^{2} \cdot f_{t}^{2}\right) /(8 R)$

Surface finish for peripheral cutting

$$
R_{a}=\left(3.18 \times 10^{2} \cdot f_{t}^{2}\right) /\left(8\left(R+N_{f} \cdot f_{t} / \pi\right)\right)
$$


Where the coefficients $K_{t}$ and $K_{r}$ indicate the ratio of tangential cutting force to the chip load, and the ratio of radial to tangential cutting force respectively.

The MLP and CMLP networks are trained off-line by using the I/O exemplars. Then, these models are tested through the checking exemplars.

An illustration of $S E_{t}, S E_{c p}$ and $S E_{c s}$ obtained through all tested neural models is presented in Fig. 2.

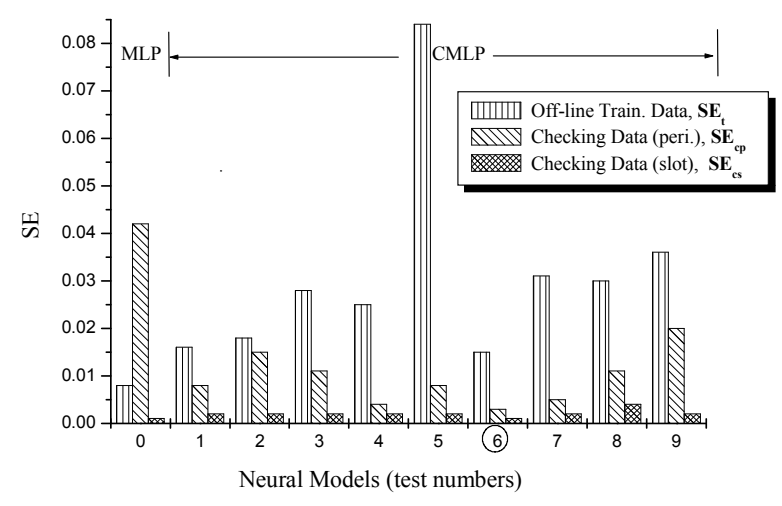

Figure 2 Graph of the flexibility simulation test

It can be observed that the CMLP network with three clusters and mixed clustering has shown minimum total error $S E_{\text {total }}$ and thus better ability to model two operations simultaneously.

\section{Reliability Simulation Test}

The reliability evaluation (i.e., the robustness as used in noisy conditions) of the proposed network architecture is based on off-line and on-line learning of the mapping existing between the control parameters and the state variable in the machining process under different machining and noise conditions.

By using the theoretical cutting parameters relationships of Eq. 1, two sets of on-line training data are simulated; first, machining data using a worn tool and second, machining data using a worn tool and noisy conditions.

The CMLP and MLP networks are just trained off-line. The sums of squared errors $\left(S E_{\text {all }, 0}\right)$ are illustrated by the first bars of Figures 3 and 4 .

It should be noted that for a clear comparison, the results of only one of the trained CMLP is shown. Every training data of the first and second sets are used to train the networks. The overall errors ( $\left.S E_{\text {all }, i}\right)$ are shown in Figures 3 and 4.

As illustrated in these figures, the MLP shows slightly better performance than the CMLP during off-line training. However, as on-line training processes the degradation of previously learned knowledge is observable so the overall errors of MLP increase. During on-line training the CMLP reveals good results and the overall errors decrease.

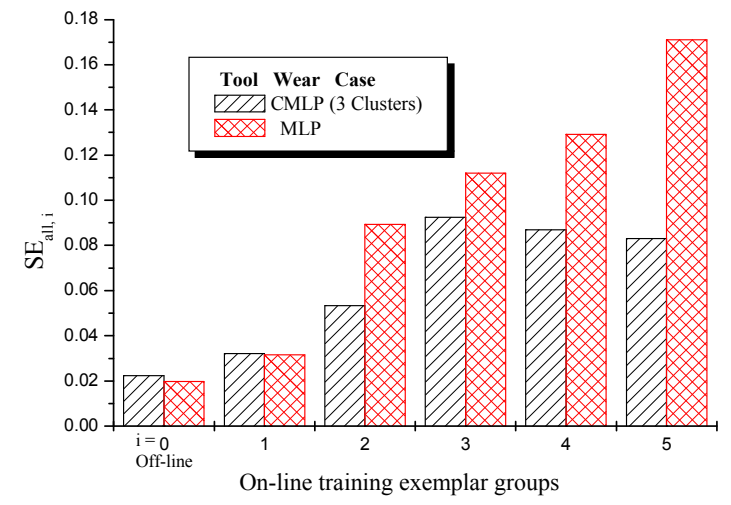

Figure 3 Results with tool wear

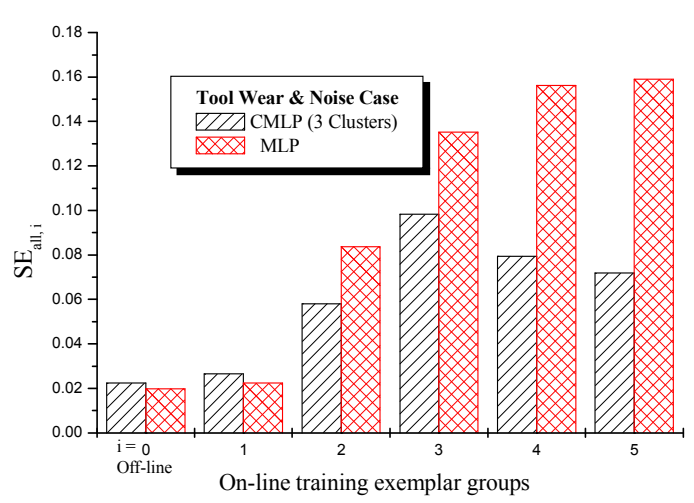

Figure 4 Results with tool wear and noise 


\section{Feedforward inverse process neuro-controller}

A simple feedforward inverse process neuro-controller as shown in Fig. 5 is now used. In this scheme adaptation to process variation is achieved with on-line neuro-controller training. The objective of the neuro-controller is to determine the optimal process input (i.e. feed) that regulates the process output (i.e. cutting force) to the desired quantity given certain perturbations.

The results obtained from the inverse neuro-controller scheme tested with (on-line training) or without adaptation are depicted in Figures 6 and 7 respectively for force and feed estimations.

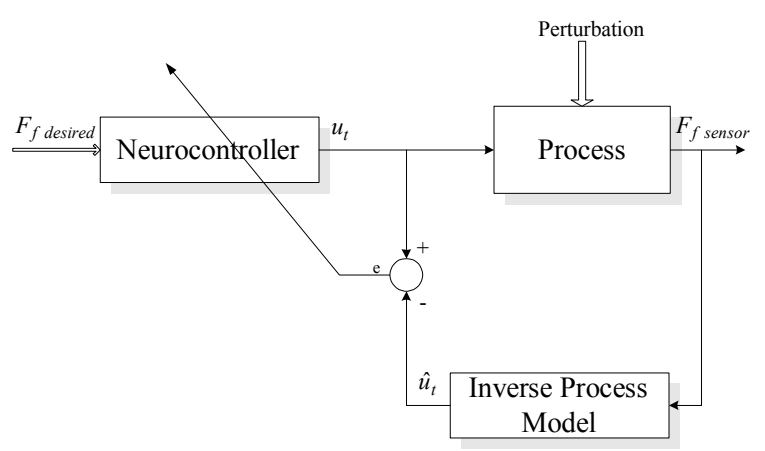

Figure 5 Feedforward adaptive inverse neuro-controller

In conclusion, the performance of the neuro-controller is much improved when its neural model is adapted on-line (c.f. Figures $6 \mathrm{~b}$ and $7 \mathrm{~b}$ ). This adaptation is realized by carrying out 10 on-line training iterations every control step. However, the control system can learn and compensate for process variations (i.e., perturbation) and therefore can provide better modeling and control.

\section{Conclusions}

On the basis of a machining case study, it was shown via simulation that the CMLP network possesses an excellent capacity to store information very locally while maintaining a satisfactory generalization. During on-line learning of new exemplars, the CMLP has demonstrated minimum information loss of previously learned knowledge.

The adaptive neuro-controller was able to compensate for the modeling errors, thus providing even better modeling and control under process variations (i.e. perturbations). This neuro-control approach can also be easily implemented in the optimization of other manufacturing processes.

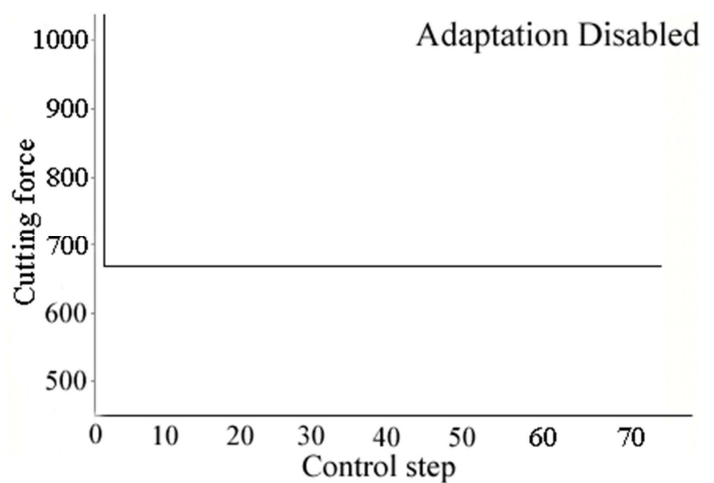

(a)

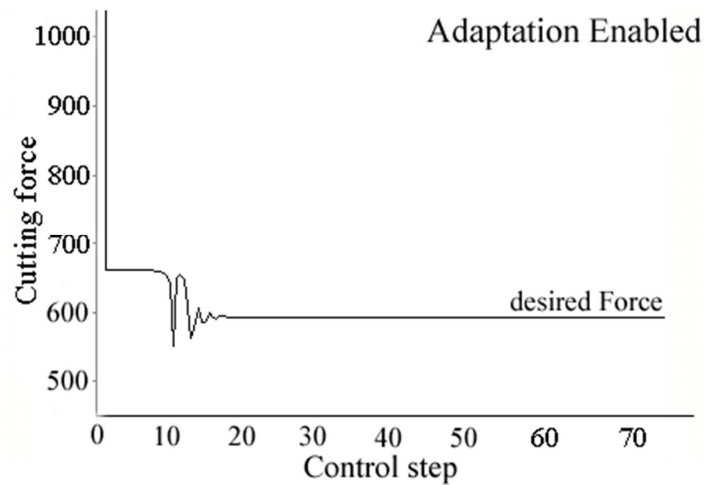

(b)

Figure 6 Output using the inverse neuro-controller (a) without adaptation and (b) with adaptation 


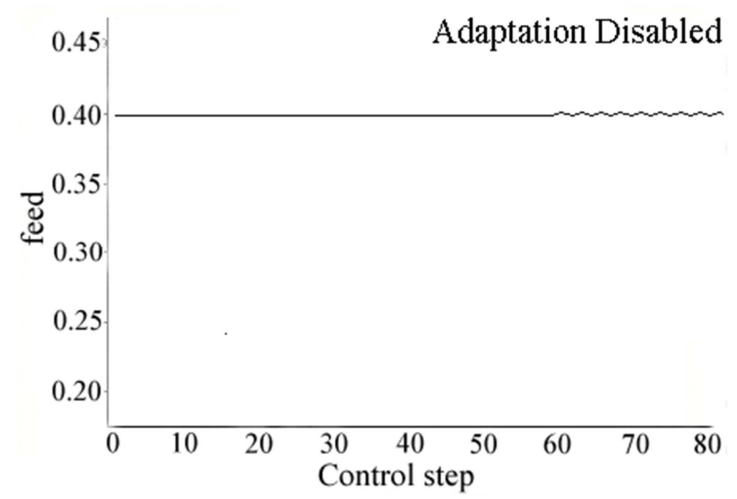

(a)

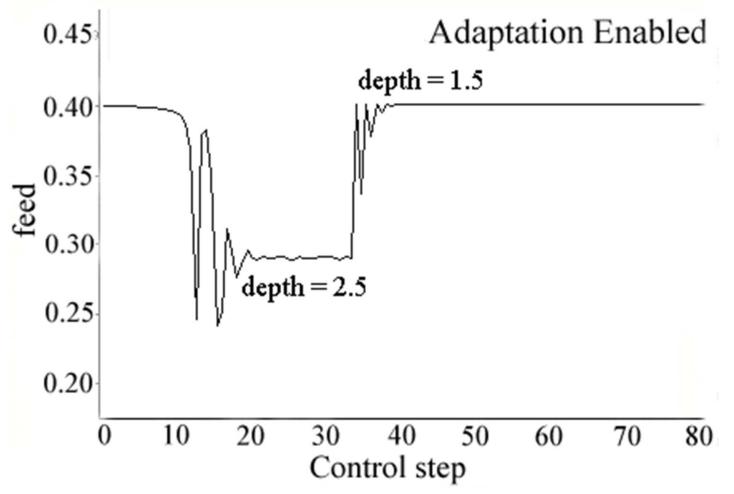

(b)

Figure 7 Process input using the inverse neuro- controller with (a) adaptation disabled and (b) adaptation enabled

\section{References}

[1] Kim S. and Klamecki B. E., "Milling Cutter Wear Monitoring using Spindle Shaft Vibration', ASME J. of Manufacturing. Science and Engineering, Vol. 119, pp. 118-119, Feb. 1997.

[2] Chryssolouris G. and Guillot M., "A Comparison of Statistical and AI Approaches to the Selection of Process Parameters in Intelligent Machining', ASME J. Eng. for Ind., Vol. 112, pp. 122-131, May 1990.

[3] Azouzi R. and Guillot M., "Study of an Inverse Process Neuro-controller for Machining Optimization”, Smart Eng. Sys. Design, Vol. 00, pp.1-12, 1998.

[4] Azouzi R. and Guillot M., "Control and Optimization of the Turning Process using a new Hybrid Neural Network", Proceedings of the Japan/USA Symposium on Flexible Automation, Boston, MA., USA, Vol. 2, July 7-10 1996.

[5] Chiang Shiuh-Tarng et al., "Adaptive Control Optimization in End Milling using Neural Networks", Int. J. Mach. Tools Manufacture., Vol. 34, No. 5, pp. 637-660, 1995.

[6] Werbos P. J., "Backpropagation and Neuro-control: A Review and Prospectus", IEEE (cat N 89CH2765-6), Piscataway, NJ, pp. 209-216, 1989.

[7] G. K. M. Raoa, G. Rangajanardhaab, D. H. Raoc, M. S. Raoa, Development of hybrid model and optimization of surface roughness in electric discharge machining using artificial neural networks and genetic algorithm, materials processing technology, x x x, 2008, xxx-xxx

[8] M. Sen, H.S. Shan, Electro jet drilling using hybrid NNGA approach, Robotics and ComputerIntegrated Manufacturing, 23, 2007, 17-24.

[9] D. Umbrello, G. Ambrogio, L. Filice, R. Shivpuri, A hybrid finite element method-artificial neural network approach for predicting residual stresses and the optimal cutting conditions during hard turning of AISI 52100 bearing steel, Materials and Design, 29, 2008, 873-883.

[10] U. Zuperla, F. Cusa, B. Mursecb, T. Ploj, A hybrid analytical-neural network approach to the determination of optimal cutting conditions, Materials Processing Technology, 157-158, 2004, 8290 . 